\title{
Time-dependent density-functional calculation of the stopping power for protons and antiprotons in metals
}

\author{
M. Quijada, ${ }^{1,2}$ A. G. Borisov, ${ }^{2,3}$ I. Nagy, ${ }^{2,4}$ R. Díez Muiño, ${ }^{2,5}$ and P. M. Echenique $e^{1,2,5}$ \\ ${ }^{1}$ Departamento de Física de Materiales, Facultad de Químicas UPV/EHU, Apartado 1072, 20080 San Sebastián, Spain \\ ${ }^{2}$ Donostia International Physics Center DIPC, P. Manuel de Lardizabal 4, 20018 San Sebastián, Spain \\ ${ }^{3}$ Laboratoire des Collisions Atomiques et Moléculaires (UMR CNRS-Université Paris-Sud 8625), Bât. 351, Université Paris-Sud, \\ 91405 Orsay Cedex, France \\ ${ }^{4}$ Department of Theoretical Physics, Institute of Physics, Technical University of Budapest, H-1521 Budapest, Hungary \\ ${ }^{5}$ Unidad de Física de Materiales, Centro Mixto CSIC-UPV/EHU, P. Manuel de Lardizabal 3, 20018 San Sebastián, Spain
}

(Received 8 September 2006; published 20 April 2007)

\begin{abstract}
Time-dependent density-functional theory is used to calculate the energy loss of antiprotons and protons traversing metal clusters of variable size. We find that the effective energy loss per unit path length inside the cluster shows no significant cluster size effects over the wide range of projectile velocities studied. This allows us to compare the calculated stopping power with the experimental values for a solid metal target. Excellent agreement between the theoretical results and recent experimental data is found for velocities below the inner-shell excitation threshold. We thus present a nonperturbative quantum-mechanical approach to obtain the energy loss of charges in solids.
\end{abstract}

DOI: 10.1103/PhysRevA.75.042902

PACS number(s): 34.50.Bw, 78.70.-g, 71.10.Ca, 71.15.Mb

\section{INTRODUCTION}

Charged particles penetrating solid media give rise to electronic excitations in the target and thus lose kinetic energy. The slowing down of charged particles in matter is a key phenomenon in applied materials science, in medical physics, as well as being an important ingredient in many experimental techniques used in fundamental research on solids, surfaces, and nanostructures. However, and despite numerous attempts, the complexity of the dynamic interaction between charges and solids has made it difficult to apply theoretical schemes at the level of accuracy achieved in other condensed matter problems. For such accuracy, a detailed description of electronic excitations, dynamic screening, and possible charge transfer processes is required.

So far, self-consistent calculations for the slowing down of ions in metal targets have been reported only in the lowvelocity limit. A combination of scattering theory with the results of static density functional theory (DFT) can successfully describe the energy loss in this case [1-5]. For higher projectile velocities, only model calculations based on velocity-dependent screening [6,7] or perturbative expansions in terms of screened higher-order response functions [8] are available at present. There has been no quantal selfconsistent theoretical framework able to describe the stopping power of solids for charged projectiles over a wide range of velocities. Only theories based on classical mechanics $[9,10]$ have been applied. Particularly challenging is the case in which the projectile velocity is similar to the Fermi velocity $v_{F}$ of the target electrons. In this velocity range, quasistatic or perturbative approximations break down even for unit-charge projectiles.

The development of time-dependent methods in recent years has opened new perspectives in the theoretical description of the slowing down of charged projectiles in matter. In particular, time-dependent density functional theory (TDDFT) provides a self-consistent, nonperturbative, time- domain treatment of electron dynamics in many body systems. TDDFT has been successfully applied to the calculation of energy transfer in collisions between charged atomic particles and small molecules and clusters in the gas phase [11-14]. Two-dimensional targets of finite size [15] and metal surfaces [16] have been addressed as well.

In this article, we develop a quantal method based on TDDFT in order to obtain the contribution of the valence band electrons to the slowing down of charges in solids over a wide range of velocities. The stopping power of $\mathrm{Al}$ for antiprotons is taken as an illustration to demonstrate the capabilities of the method. There are several reasons for this choice. First, the valence electrons of $\mathrm{Al}$ are the paradigm of a free-electron-like band, so that numerical effort can be much reduced. Second, pointlike projectiles of negative charge have often been under scrutiny since the pioneering paper of Fermi and Teller [17]: The absence of charge exchange processes between the projectile and the target simplifies the analysis in this case. Finally, accurate experimental data have recently been obtained for this system [18-20]. It is worth mentioning that our method is not limited to freeelectron-like targets: The methodology is general and could be in principle applied to any weakly correlated material.

The core of our method consists of TDDFT calculations of the energy loss of projectiles traversing target clusters of variable sizes. We then show that the energy loss per unit path length inside the cluster is nearly independent of the cluster size. This allows us to link this quantity to the stopping power of an infinite system. Our theoretical results for the stopping power of $\mathrm{Al}$ for antiprotons reproduce recent experimental measurements [18-20] for the range of projectile velocities below the inner-shell excitation threshold. Extension of the present approach to the stopping of $\mathrm{Al}$ for protons agrees quantitatively with experimental data $[19,20]$ as well. 


\section{THEORETICAL APPROACH}

To calculate the stopping power of a cluster of $\mathrm{Al}$ atoms, a spherical jellium model is used to represent the valence electrons. The contribution of inner-shell excitations to the stopping is thus not included. The jellium positive background density is defined by $n_{0}^{+}(\mathbf{r})=n_{0}^{+} \theta\left(R_{\mathrm{cl}}-r\right)$. Here, $R_{\mathrm{cl}}$ is the cluster radius and $\theta(x)$ is the Heaviside function. The electron density of the cluster is described by the density parameter $r_{s}\left(4 \pi r_{s}^{3} / 3=1 / n_{0}\right)$. The number of electrons in the cluster $N_{e}$ is $N_{e}=\left(R_{\mathrm{cl}} / r_{s}\right)^{3}$.

The time evolution of the electron density in response to the field of the moving projectile, $n(\mathbf{r}, t)$, is calculated within the Kohn-Sham (KS) scheme of TDDFT (Hartree atomic units are used everywhere unless otherwise stated):

$$
i \frac{\partial \psi_{j}(\mathbf{r}, t)}{\partial t}=\left\{T+V_{\mathrm{eff}}([n], \mathbf{r}, t)\right\} \psi_{j}(\mathbf{r}, t),
$$

where $\psi_{j}(\mathbf{r}, t)$ are the KS orbitals and $T$ is the kinetic energy operator. The effective KS potential, $V_{\text {eff }}([n], \mathbf{r}, t)$ is a function of the electron density of the system: $n(\mathbf{r}, t)$ $=\sum_{j \in \text { occ }}\left|\psi_{j}(\mathbf{r}, t)\right|^{2}$. Here, $V_{\text {eff }}$ is obtained as the sum of the external potential $V_{\text {ext }}$, the Hartree potential $V_{H}$, and the exchange correlation potential $V_{\mathrm{xc}}: V_{\mathrm{eff}}=V_{\mathrm{ext}}+V_{H}+V_{\mathrm{xc}} \cdot V_{\mathrm{ext}}$ is the Coulomb potential created by a point charge $Q$ moving with constant velocity $v$ along a straight trajectory that goes through the geometrical center of the cluster. The initial position of the projectile is such that the projectile-cluster interaction can be neglected. $V_{\mathrm{xc}}(\mathbf{r}, t)$ is treated in a standard adiabatic local density approximation (ALDA) with the exchange-correlation functional of Ref. [21].

The numerical procedure used is very similar to that of Refs. [15,22]. For $t=0, n(\mathbf{r}, 0)$ is the electron density of the unperturbed cluster. The KS wave functions $\psi_{j}(\mathbf{r}, 0)$ are expanded in a basis set of spherical harmonics. A radial mesh of equidistant points is used, allowing the Fourier grid representation of the Hamiltonian matrix [23]. The static KS equations are then solved by direct diagonalization. Afterwards, the $\mathrm{KS}$ orbitals are projected onto a cylindrical grid, $\mathbf{r}=(\varrho, \varphi, z)$, with the $z$ axis along the projectile trajectory. The time propagation is performed by means of the splitoperator technique [24].

\section{RESULTS AND DISCUSSION}

Figure 1 shows several snapshots of the change in electron density $\Delta n(\mathbf{r}, t)=n(\mathbf{r}, t)-n(\mathbf{r}, 0)$ induced by an antiproton $(Q=-1)$ of velocity $v=1.5$ colliding with a cluster of $N_{e}=254, R_{\mathrm{cl}}=12.7$, and $r_{s}=2$. This value of $r_{s}$ is close to that describing the screening radius in bulk Al. The density is plotted in a plane that contains the particle trajectory. A polarization effect is clearly visible before the antiproton reaches the cluster surface. Once inside the cluster, a depletion of charge develops in the vicinity of the antiproton. The self-consistent dynamic rearrangement of charge in the vicinity of the moving particle results in a wake potential similar to that in bulk metal targets [25]. Our results show that the collision of the antiproton with the cluster is followed by electron emission from the cluster.
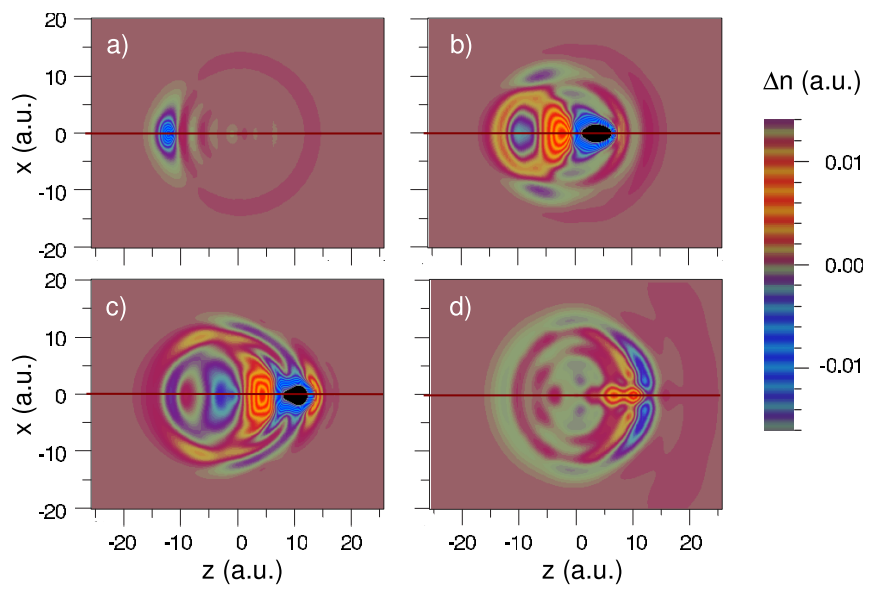

FIG. 1. (Color online) Contour plots for the antiproton-induced change $\Delta n(\mathbf{r}, t)$ in the electron density of the spherical cluster. The cluster parameters are $r_{s}=2$ and $N_{e}=254$. Results are shown in the $(x, z)$ plane. The center of the cluster is at $(0,0)$. The projectile trajectory is shown as a horizontal line in each plot. The projectile velocity is $v=1.5$. Plots (a) to (d) correspond to antiproton positions $z=-12.7,5.8,12.6$, and 42.5. Color codes are shown at the right side of the figure.

Figure 2 shows the force acting on the antiproton during the collision process. It is directly obtained from the timedependent charge density, $n_{0}^{+}(\mathbf{r})-n(\mathbf{r}, t)$. Due to the cylindrical symmetry of the problem, the only nonzero component of the force is along the projectile trajectory ( $z$ axis), $F_{z}(t)$. When the antiproton is located outside the cluster, it is attracted by the induced dipole. Crossing the surface into the cluster results in a rapid rearrangement of electron density to screen the projectile charge. Inside the cluster, $F_{z}$ shows minor oscillations around a constant (mean) value.

The energy lost by the projectile during the collision is given by

$$
\Delta E=v \int_{-\infty}^{\infty} F_{z}(t) d t
$$

We have explicitly checked that $|\Delta E|$ calculated from Eq. (2) corresponds to the increase of the total energy of the cluster. However, the magnitude we are interested in is the effective stopping power inside the cluster $S$. For this purpose, we

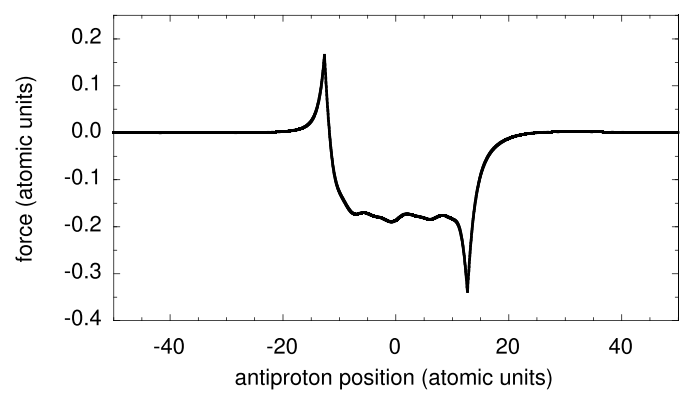

FIG. 2. Force on an antiproton moving with $v=1.5$ as a function of its position with respect to the center of the cluster. The cluster parameters are $r_{s}=2, N_{e}=254$, and $R_{\mathrm{cl}}=12.7$. 


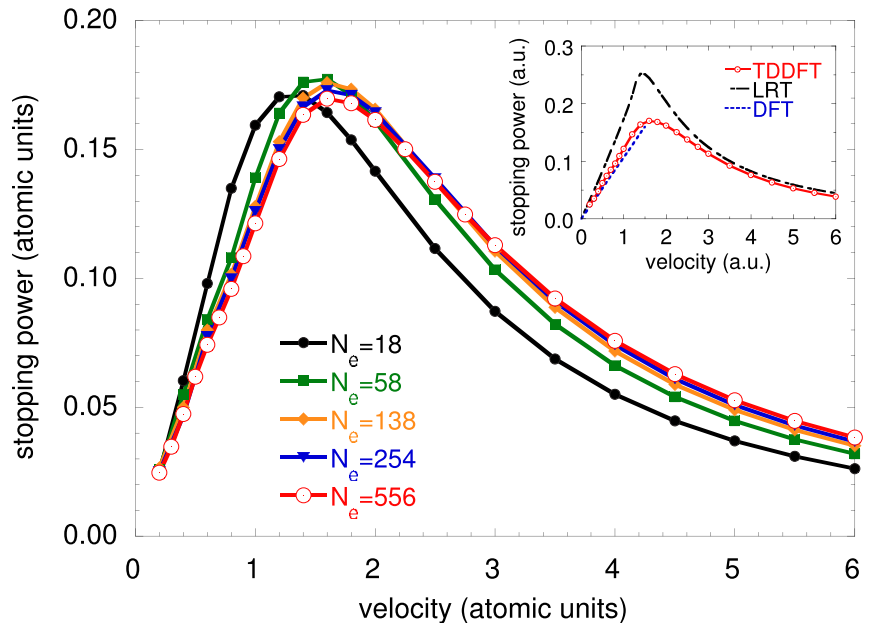

FIG. 3. (Color online) Stopping power $S$ of antiprotons moving inside clusters with $r_{s}=2.07$ and of different sizes (see legend), as a function of velocity. Lines are drawn to guide the eye. The inset compares the $N_{e}=556$ results with those of LRT and DFT.

define $S$ as the ratio of the energy loss to the trajectory length inside the cluster, i.e., the diameter of the cluster: $S$ $=\Delta E /\left(2 R_{\mathrm{cl}}\right)$.

Figure 3 shows $S$ as a function of projectile speed for antiprotons colliding with clusters of $r_{s}=2.07$ and size ranging from $N_{e}=18\left(R_{\mathrm{cl}}=5.42\right)$ to $N_{e}=556\left(R_{\mathrm{cl}}=17.02\right)$. The valence electrons of bulk $\mathrm{Al}$ can be well represented by this value of $r_{s}$. Size effects are only significant for the two smallest clusters considered $\left(N_{e}=18\right.$ and $\left.N_{e}=58\right)$. For the largest ones, the results merge roughly into a universal curve. We estimate that deviations from this universal behavior for larger clusters amount to a maximum of $\approx 5 \%$. The similarity of the results for different clusters reflects important features of the dynamics of the interaction within the studied velocity range: (i) The contributions to $\Delta E$ from the ingoing and outgoing trajectory paths, as well as from the surface region, are small. Most of the energy loss is suffered while moving inside the cluster. (ii) The size independence suggests that the dynamic screening within the cluster is essentially that for a homogeneous system in the velocity range considered. This implies that the discreteness of electronic states in the cluster does not play an important role in the screening process.

The independence of the value of $S$ on the cluster size allows us to consider $S$ as representative of the stopping power in an infinite free electron gas with the same electron density parameter $r_{s}$. In the inset of Fig. 3, we compare our TDDFT results with other theoretical approximations for the infinite system, which are known to be accurate under more restricted conditions. The linear response theory (LRT) result provides the high velocity limit. It is obtained using a Mermin linear response function [26] with an empirical damping factor of $\gamma=1.35 \mathrm{eV}$. The DFT result [2] assumes a linear dependence of $S$ on $v$ and provides the low-velocity limit. Both the low-velocity limit and the high-velocity limit for the infinite system are well-described by our calculation.

In Fig. 4, we compare our TDDFT results for the $N_{e}$ $=556$ cluster with experimental measurements recently reported for bulk Al [18-20]. The TDDFT results quantita-

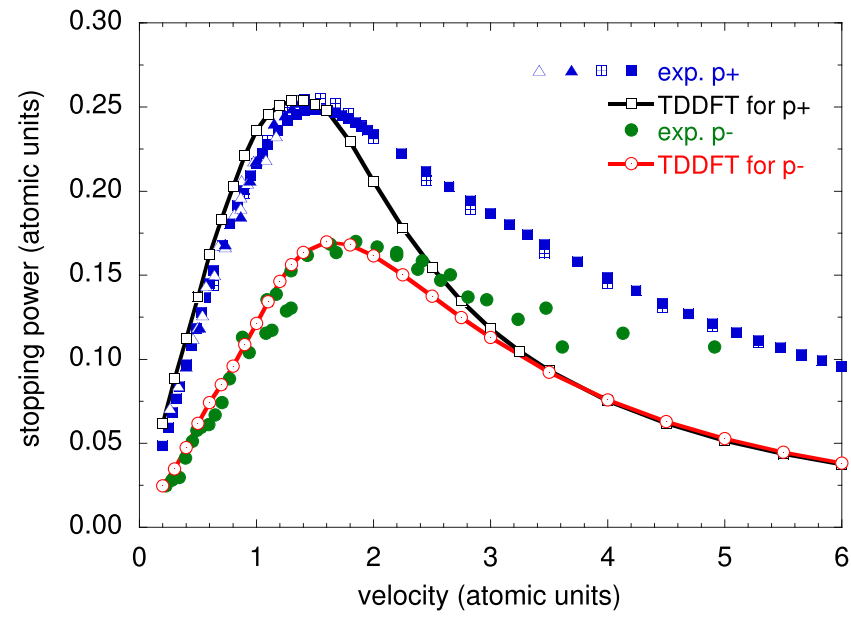

FIG. 4. (Color online) TDDFT calculation of the stopping power $S$ of Al ( $\left.r_{s}=2.07\right)$ for protons (black empty squares) and antiprotons (red empty circles) as a function of projectile velocity. Experimental results for antiprotons (green filled circles [18-20]) and protons (blue filled and empty triangles [20]), as well as tabulated stopping powers for protons (blue crossed [27] and filled [28,29] squares) are also shown.

tively agree with the experimental data, up to velocities beyond the stopping power maximum. Deviations arise at $v$ $\approx 1.8$, when the excitation of the $\mathrm{Al}$ inner-shell electrons starts to contribute to the projectile energy loss. This channel is not included in our calculation. For low-velocity projectiles, our results show a linear dependence of $S$ on $v$, roughly up to the maximum in $S$ : deviations from the linear dependence are smaller than $6 \%$ for velocities $v<1.4$.

The description of the interaction between negatively charged atomic particles and metals is simplified by the absence of charge exchange. For positive ions, and particularly for velocities lower than $v_{F}$, electron capture and loss processes come into play. In order to test the accuracy of the TDDFT-ALDA model for positive ions, we have performed additional calculations for the stopping of $\mathrm{Al}$ for protons $(Q=+1)$. The procedure is identical to that used for antiproton projectiles. The results are shown in Fig. 4. They are compared with recent experimental measurements [19,20]. Reference tabulations of stopping ranges in solids are shown as well [27-29]. The agreement is good up to the velocity for which the excitation of the Al inner shells starts to contribute. According to our TDDFT result, the difference between the stopping power for protons and that for antiprotons due to the excitation of the $\mathrm{Al}$ valence band is small $(<10 \%)$ for $v \geqslant 3$, suggesting a small valence Barkas effect.

\section{SUMMARY AND CONCLUSIONS}

In summary, we conclude that finite sized systems can be used to study the energy loss of charged projectiles moving inside metallic solids. The local character of the interaction makes it possible to define an effective stopping power that is shown to be comparable to that of an infinite target. The specific example of the free-electron metal is treated here, but the methodology developed in the present work is gen- 
eral. It can be applied to any material for which the electronic structure can be well characterized within a DFT framework. Further studies should be made to investigate whether our conclusions are also applicable to semiconductors and insulators, where the adiabatic approximation for correlations may also have limitations [30].

Furthermore, we have shown that TDDFT is a promising approach for the calculation of the mean energy loss of point charges in matter. It is a quantal, nonperturbative method, which is able to describe projectile-target energy transfer over a wide range of projectile velocities. The support for this statement is twofold: (a) Our TDDFT calculations reproduce stopping powers in the well-known limits of low- and high-velocity projectiles and (b) they quantitatively agree with recent data for the stopping power of $\mathrm{Al}$ for protons and antiprotons. The agreement in the case of protons is particulary interesting and worthy of more detailed analysis, as it suggests that charge transfer processes are reasonably well described within the ALDA.

\section{ACKNOWLEDGMENTS}

Financial support by the Basque Departamento de Educación, Universidades e Investigación, the University of the Basque Country UPV/EHU (Grant No. 9/UPV 00206.21513639/2001), the Spanish MEC (Grant No. FIS2004-06490C03-00), and the EU Network of Excellence NANOQUANTA (Grant No. NMP4-CT-2004-500198) is acknowledged. The work of I.N. was partially supported by the Hungarian OTKA (Grant Nos. T046868 and T049571).
[1] P. M. Echenique, R. M. Nieminen, and R. H. Ritchie, Solid State Commun. 37, 779 (1981).

[2] I. Nagy, A. Arnau, P. M. Echenique, and E. Zaremba, Phys. Rev. B 40, R11983 (1989).

[3] A. H. Sørensen, Nucl. Instrum. Methods Phys. Res. B 48, 10 (1990)

[4] A. Narmänn, R. Monreal, P. M. Echenique, F. Flores, W. Heiland, and S. Schubert, Phys. Rev. Lett. 64, 1601 (1990).

[5] A. Salin, A. Arnau, P. M. Echenique, and E. Zaremba, Phys. Rev. B 59, 2537 (1999).

[6] I. Nagy and B. Apagyi, Phys. Rev. A 58, R1653 (1998).

[7] N. R. Arista and A. F. Lifschitz, Phys. Rev. A 59, 2719 (1999).

[8] J. M. Pitarke, R. H. Ritchie, and P. M. Echenique, Phys. Rev. B 52, 13883 (1995).

[9] P. Sigmund and A. Schinner, Eur. Phys. J. D 12, 425 (2000).

[10] F. Grüner, F. Bell, W. Assmann, and M. Schubert, Phys. Rev. Lett. 93, 213201 (2004).

[11] K. Yabana, T. Tazawa, Y. Abe, and P. Bozek, Phys. Rev. A 57, R3165 (1998).

[12] U. Saalmann and R. Schmidt, Phys. Rev. Lett. 80, 3213 (1998); T. Kunert and R. Schmidt, ibid. 86, 5258 (2001).

[13] R. Baer and N. Siam, J. Chem. Phys. 121, 6341 (2004).

[14] T. A. Niehaus, D. Heringer, B. Torralva, and Th. Frauenheim, Eur. Phys. J. D 35, 467 (2005).

[15] A. G. Borisov, J. I. Juaristi, R. Díez Muiño, D. Sánchez-Portal, and P. M. Echenique, Phys. Rev. A 73, 012901 (2006).

[16] M. Lindenblatt, E. Pehlke, A. Duvenbeck, B. Rethfeld, and A. Wucher, Nucl. Instrum. Methods Phys. Res. B 246, 333
(2006).

[17] E. Fermi and E. Teller, Phys. Rev. 72, 399 (1947).

[18] S. P. Møller, E. Uggerhøj, H. Bluhme, H. Knudsen, U. Mikkelsen, K. Paludan, and E. Morenzoni, Phys. Rev. A 56, 2930 (1997).

[19] S. P. Møller, A. Csete, T. Ichioka, H. Knudsen, U. I. Uggerhøj, and H. H. Andersen, Phys. Rev. Lett. 88, 193201 (2002).

[20] S. P. Møller, A. Csete, T. Ichioka, H. Knudsen, U. I. Uggerhøj, and H. H. Andersen, Phys. Rev. Lett. 93, 042502 (2004).

[21] O. Gunnarson and B. I. Lundqvist, Phys. Rev. B 13, 4274 (1976).

[22] A. Borisov, D. Sánchez-Portal, R. Díez Muiño, and P. M. Echenique, Chem. Phys. Lett. 387, 95 (2004).

[23] R. Kosloff, J. Phys. Chem. 92, 2087 (1988).

[24] M. D. Feit and J. A. Fleck, Jr., J. Chem. Phys. 78, 301 (1982).

[25] J. Neufeld and R. H. Ritchie, Phys. Rev. 98, 1632 (1955).

[26] N. D. Mermin, Phys. Rev. B 1, 2362 (1970).

[27] H. Paul, D. Semrad, and A. Seilinger, Nucl. Instrum. Methods Phys. Res. B 61, 261 (1991).

[28] H. H. Andersen and J. F. Ziegler, Hydrogen Stopping Powers and Ranges in all Elements (Pergamon Press, Elmsford, NY, 1977).

[29] International Commission on Radiation Units and Measurements, Stopping Powers and Ranges for Protons and AlphaParticles, ICRU Report 49 (Oxford University Press, Oxford, UK, 1993).

[30] J. Tao and G. Vignale, Phys. Rev. Lett. 97, 036403 (2006). 